phytosociologique et biogéographie des groupements rupicoles calcaires du Maroc. Bull. Inst. Bot. Rabat (en prensa).

FIORI, A. y G. PAOLETTI -1904- Flora analitica d'Italia III. Padova.

GALÁN DE MERA, A. y J.A. VICENTE ORELLANA -1998- Lectotypification of Hypochaeris leontodontoides and other notes on the Hypochaeris laevigata group (Asteraceae). Taxon 47: 115-116.

LITARDIÈRE, R. y R. MAIRE-1924-Contribution à l'étude de la flore du Grand Atlas. Mém. Soc. Sci. Nat. Maroc 4: 1-32.
OBERDORFER, E. -1990- Pflanzensoziologische Excursionsflora. Stuttgart.

PIGNATTI, S. -1982- Flora d'Italia III. Bologna.

QUÉZEL, P. -1957-Peuplement végétal des hautes montagnes de l'Afrique du Nord. Paris.

Aceptado para su publicación en Junio de 1998

Dirección de los autores: Laboratorio de Botánica. Departamento de Biología. Universidad San PabloCEU. Ap. 67. E- 28660 Boadilla del Monte (Madrid). Fax: 91351049 6, E-mail: agalmer@ceu.es

\title{
59. ACERCA DE TRES ESPECIES NITRÓFILAS EN LA PROVINCIA DE ALMERÍA
}

\author{
Elías DANA, Juan F. MOTA, Javier CABELLO y Julio PEÑAS
}

Concerning three nitrofilous species in Almeria province

Palabras clave. malas hierbas, corología, Almería, España.

Key words. weeds, chorology, Almería, Spain.

Con motivo de un trabajo de investigación sobre vegetación urbana en Almería, hemos registrado la presencia de más de doscientos taxones nitrófilos. De este contingente, seleccionamos tres especies para la presente nota: Spergula fallax, Chamaesyce serpens y Atriplex semibaccata, por su particular interés biogeográfico y malherbológico. De todas ellas, sólo la primera puede considerarse autóctona. Bajo el nombre de cada taxón indicamos las coordenadas UTM de la zona de herborización y la referencia de herbario.
Spergula fallax (Lowe) E. H. L. Krause.

ALMERÍA: Almería ciudad, UTM 30SWF4979, $10 \mathrm{~m}$., cultivos abandonados alrededor de la ciudad, 15/03/97, E. Dana, Herbario de la Universidad de Almería 585.

Terófito disyunto asiático-mediterráneo occidental (incluyendo Canarias y Madeira), con desarrollo invernal y primaveral. Su presencia en el continente europeo sólo es conocida con certeza en Almería, concretamente en dos puntos distantes entre sí unos $60 \mathrm{Km}$, los Campos de Dalías y la comarca 
del Cabo de Gata (Ratter in Castroviejo et al., 1990). Sin embargo, hemos localizado varias poblaciones en el límite norte del casco urbano de la capital; este punto, casi equidistante de los anteriores, está caracterizado por la abundancia de cortijos y cultivos abandonados desde hace al menos 20 años. Debido a la intensa antropización a que ha estado sometida la zona desde hace pocas décadas, algunas poblaciones detectadas se encuentran degradadas y frecuentemente entremezcladas con especies propias de medios ruderalizados, si bien algunas otras parecen mantener su autoecología típica (pastos subnitrófilos semiáridos).

La actual distribución de la especie en Almería, y el hecho de que el tipo de ambientes en los que habita esta especie abunden en la provincia, nos induce a pensar que probablemente la distribución de este interesante taxón en la provincia sea algo mayor de lo que actualmente se cree. Por el contrario, en el ambiente urbano gran parte de las poblaciones han debido ser destruidas conforme se ha ido expandiendo la ciudad; por tratarse de un paraje que sufrirá una próxima urbanización, se ha procedido a la recolección y conservación de propágulos en el banco de germoplasma de la Universidad de Almería.

\section{Chamaesyce serpens (Kunth) Small}

ALMERÍA: Almería ciudad, UTM 30SWF4877, $10 \mathrm{~m}$., cultivos abandonados alrededor de la ciudad, 10/06/94, E. Dana, Herbario de la Universidad de Almería, 586; Campos de El Ejido, 100m, terrenos alterados, 10/04/94, G. Bonilla \& J. Mota, Herbario de la Universidad de Almería 589.

Pequeño terófito postrado de origen norteamericano. Detectamos su presencia en 1994, en ambientes antropizados de la comarca del Poniente almeriense. Ese mismo año fue observada creciendo abundantemente en numerosos jardines y parterres de la ciudad de
Almería. Desde entonces hemos realizado un seguimiento de la evolución de la especie para comprobar si su presencia se mantiene, ya que se han descrito casos de «agresividad» en algunas especies similares; este es el caso de Chamaesyce nutans, en el Valle del Guadalquivir (Hernández-Bermejo et al., 1984). Hemos podido apreciar que se han producido varias reintroducciones de la especie mediante la tierra que acompaña a los árboles ornamentales plantados en la ciudad, por lo que parece obvio el mecanismo de expansión en la provincia, especialmente, si se tienen en cuenta las citas de esta especie en otras provincias del Levante con las que Almería mantiene intercambio comercial de especies ornamentales.

Su existencia ha sido detectada también en otras zonas del litoral mediterráneo peninsular como Murcia (Alcaraz, 1984), la Comunidad Valenciana (Mateo y Crespo, 1990) y Cataluña (Bolòs et al., 1993), así como en el Valle del Guadalquivir y la mayor parte de Cádiz (Valdés in Valdés et al., 1987). En la segunda mitad de este siglo, Smith \& Tutin (in Tutin et al., 1968) afirmaron que en Europa se encontraba en aquellos momentos naturalizándose en algunos puntos de España y en el Sur de Francia. Todo ello indica que este taxón está experimentando una clara expansión en España.

Además de Europa, ha sido introducida en otros lugares del mundo, como Uruguay y Argentina, donde es utilizada como diurética, motivo por el que es conocida como «yerba meona» (Dimitri in Dimitri et al., 1988).

Atriplex semibaccata $\mathrm{R}$. Br.

ALMERÍA: Almería ciudad, UTM 30SWF4977, 10 m., solares y cultivos abandonados alrededor de la ciudad, 25/08/95, E. Dana, Herbario de la Universidad de Almería 656; Los Millares (Santa Fé de Mondújar), UTM.30SWF4391, 300m, terrenos alterados por excavación arqueológica, 10/04/97, J. Peñas, Herbario de la Universidad de Almería 587. 
Hemicriptófito sufruticoso y decumbente, originario de las zonas más cálidas de Australia. Ha sido introducida en áreas de clima mediterráneo cálido como despensa casi permanente de forraje debido a su carácter perenne, a la facilidad con que rebrota y a su tolerancia frente a suelos salinos. Este es el caso de Chile (Montenegro et al. in Groves \& Di Castri, 1991) o Argentina (Dimitri in Dimitri et al., 1987). Es considerada por Le Houérou (1991), una de las especies más importantes entre las pocas alóctonas que han tenido un impacto significativo en los ambientes mediterráneos donde ha sido introducida.

Una de las primeras citas fuera de su región de origen se debe a Trabut (1904), quien la localizó en Tunicia en 1895. Maire (1962) la cita para el mismo país indicando que está naturalizada. Quézel \& Santa (1962) la recogen como cultivada y a veces asilvestrada en Argelia.

Su existencia en la provincia fue indicada por primera vez por Kunkel (1982), quién la propuso como primera cita provincial y europea, aunque sin referencia de herbario; quizá por ello en obras posteriores se ha obviado la presencia de esta especie en la provincia. En cuanto a otras zonas del litoral mediterráneo peninsular, Castroviejo (in Castroviejo et al., 1990) la considera como subespontánea en Murcia y Alicante. Mateo y Crespo (op.cit.) la citan para Alicante y Valencia. En cuanto a la provincia de Murcia, Sánchez-Gómez et al. (1986) la recogen para Murcia y Lorca.

Realizamos un seguimiento de su presencia en la ciudad y proximidades desde 1986, y hemos observado que crece de forma abundante en solares y escombreras poco perturbados así como en cultivos abandonados y taludes, casi siempre sobre suelos algo salinos. En 1997 las poblaciones de esta especie se extienden ya hasta unos $5 \mathrm{Km}$ al este de la ciudad. El abandono de los cultivos aledaños, parece ser responsable parcial de la progresión de esta especie hacia Levante, ya que

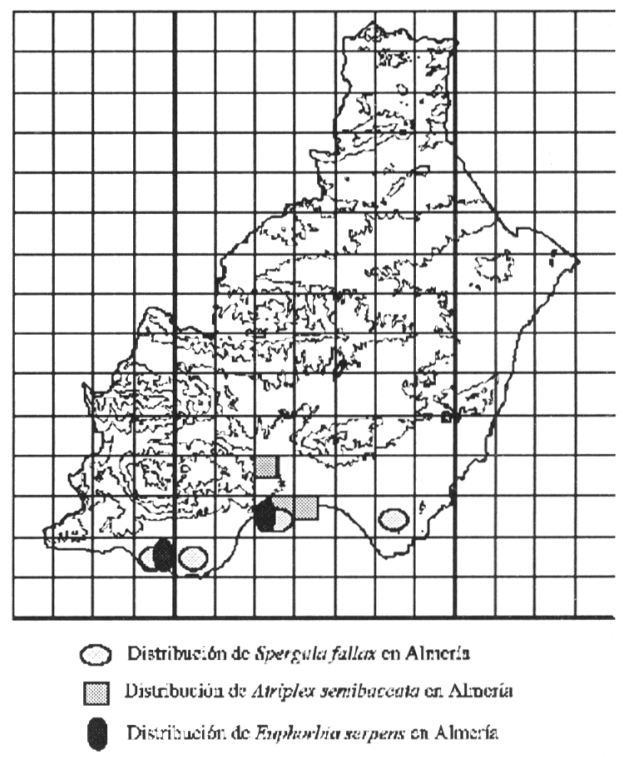

Figura 1. Distribución de las especies estudiadas. Distribution of the studied species.

proporcionan un hábitat adecuado y extenso. Además, en verano de 1997 la localizamos en las inmediaciones del yacimiento arqueológico de «Los Millares», en el término municipal de Santa Fé de Mondújar (Almería). Todo parece indicar que, al igual que en el caso anterior, se trata de una especie cuya área de distribución esta en clara expansión.

Debemos hacer notar que las zonas donde se recoge por primera vez la presencia de las dos especies alóctonas de la presente nota, $E$. serpens y A. semibaccata, están en la franja litoral de la provincia. Esto reafirma las observaciones realizadas por Masalles et al. (1996), acerca del importante papel que juegan las comarcas litorales que superan una cierta densidad de población, como punto de introducción y origen de colonización de especies alóctonas.

Por otro lado, hay que recordar, tal y como indica Guillerm (in Groves \& Di Castri, 1991) que algunas especies cuando son 
introducidas, necesitan formar poblaciones de un tamaño mínimo antes de que se manifieste su carácter invasivo; mientras tanto, quedan acantonadas en biotopos ruderales, donde «aguardan» una oportunidad que les permita aumentar su número y comenzar a expandirse hacia los campos y hábitats cercanos. Esta estrategia ha sido observada en distintas especies, como Senecio inaequidens (Guillerm et al. in Di Castri et al., 1990). Ya que, como se ha visto, las citas de $A$. semibaccata en España se concentran principalmente en ciudades y entornos alterados próximos, ésta podría ser la estrategia seguida por esta especie. Por ello es recomendable un seguimiento en el futuro. Forcella (1985) sugiere que las velocidades de expansión de especies de malas hierbas que aún no han alcanzado su distribución final puede ser utilizadas como método para predecir la expansión de futuras invasiones también para especies con requerimientos ecológicos similares. MacWorter \& Chandler (in Charudattan \& Walker, 1982) también resaltan la importancia del conocimiento del ritmo y patrones de expansión para garantizar el éxito de las estrategias de control. En este sentido, destaca la especie mencionada por tratarse de una especie introducida recientemente y que está en vías de expansión.

El actual área de distribución en la provincia de Almería de los taxones tratados se observan en el mapa adjunto (fig. 1).

\section{BIBLIOGRAFÍA}

ALCARAZ, F. -1984- Flora y Vegetación del N.E. de Murcia. Secr. Publ. Univ. Murcia. Murcia.

BOLÒS, O., J. VIGO, R. MASALLES y J. NINOT -1993- Flora Manual dels Països Catalans. Ed. Barcino.

CAstroviejo, S. -1990-Atriplex Cent. En Castroviejo, S., Laínz, M., López-González, G., Monserrat, P., Muñoz-Garmendia, F., Paiva, J. \& Villar, L. (eds.), Flora Ibérica, vol. II. Real Jardín Botánico de Madrid. C.S.I.C.
DIMITRI, H.J. -1987- Atriplex L. En Dimitri, H.J., Burkart, A., Costa de Oliveri, C., La Porte, J., Correa de Boelcke, Fescher, H., Pingitore, E. \& Biloni, J. (eds.), Enciclopedia Argentina de Agricultura y Jardinería. Ed. Acme S.A.C.I. Argentina.

DIMITRI, M.J. -1988-Euphorbia L. En Dimitri, M.J., Costa de Oliveri, C., Fechser, H., Santos, J. \& Holna, H.(eds.), Enciclopedia Argentina de Agricultura y Jardinería. Ed. Acme S.A.C.I. Argentina.

LE HOUÉROU, H.N. -1991- Plant invasions in the rangelands of the isoclimatic mediterranean zone. En Groves, R.H. \& Di Castri, F. (eds.), Biogeography of Mediterranean Invasions. Cambridge University Press.

FORCELLA, F. - 1985- Final distribution is related to rate of spread in alien weeds. Weed Research 25: 181-191.

GUILLERM, J. L. -1991- Weed invasions in agricultural areas. En Groves, R.H. \& Di Castri, F. (eds.), Biogeography of Mediterranean Invasions. Cambridge University Press.

GUILLERM, J.L., E. LE FLOC'H, J. MAILLET \& C. BOULET -1990- The invading weeds within the western Mediterranean Basin. En Di Castri, F., Hansen, A.J. \& Debussche, M. (eds.) Biological Invasions in Europe and the Mediterranean Basin.

HERNÁNDEZ-BERMEJO, J.E., M. SAAVEDRA, B. HIDALGO, J. MONTERO y L. GARCÍATORRES -1984- Weed flora in the irrigated crops of the Guadalquivir River valley. Euphorbia nutans Lag., a new weed species. Proc. of 3rd. International Symposium of E.W.R.S. on Weed Problems in the Mediterranean Area. Oeiras, Portugal 3: 621-628.

KUNKEL, G. -1982- Sobre Periploca angustifolia y notas sobre algunas plantas forasteras en la flora de Almería, España. Paralelo 37 6: 53-57.

MACWORTER, C. \& CHANDLER, J.M. -1982Conventional weed control technology. En Charudattan, R. \& Walker, H.L. (eds.) Biological Control of Weeds with Plant Pathogens. Nwe York: Wiley.

MAIRE, R. -1962- Flore de l'Afrique du Nord. Editions. Paul Lechavalier.

MASALLES, R.M., F.X. SANS y J. PINO -1996Flora alóctona de origen americano en los cultivos de Cataluña. Anales Jard. Bot. Madrid 54 (1): 436-442. 
MATEO, G. y M.B. CRESPO -1990-Claves para la Flora Valenciana. Ed. Del Cenia al Segura. MONTENEGRO, G., S. TEILLIER, P. ARCE \& V. POBLETE - 1991- Introduction of plants into the mediterranean-type climate area of Chile. En Groves, R.H. \& F. Di Castri (eds.), Biogeography of Mediterranean Invasions. Cambridge Univesity Press.

QUÉZEL, P. \& S. SANTA -1962- Nouvelle Flore de l'Algérie et des régions désertiques méridionales, vol. I. Ed. Centre National de la Recherche Scientifique. París.

RATTER, J.A. -1990- Spergula L. En Castroviejo, S., Laínz, M., López-González, G., Monserrat, P., Muñoz-Garmendia, F., Paiva, J. \& Villar, L. (eds.), Flora Ibérica, vol. II. Real Jardín Botánico de Madrid. C.S.I.C.

SMITH, J. \& T.H. TUTIN - 1968-Euphorbia L. En Tutin, T.H., Heywood, V., Burguess, N., Moore, D., Valentine, D., Walters, S. \& Webb, D. (eds.) Flora Europaea, vol. 2.
TRABUT, L. - 1904- Naturalisation de deux Atriplex australiens dans le Nord de'l Afrique $(A$. halimoides Lindl., A. semibaccata R.Br.). Bull. Société Bot. France 51: 105-106.

VALDÉS, B. -1987- Euphorbia L. En VALDÉs, B., Talavera, S. \& Galiano, E. (eds.)Flora Vasular de Andalucía Occidental, vol. 2.

Aceptado para su publicación en Junio de 1998

Dirección de los autores. Dpto. de Biología Vegetal y Ecología. Universidad de Almería. E04120Almería. España.

\title{
60. APORTACIONES A LA FLORA VASCULAR DE SIERRA NEVADA (ALMERÍA): EL ELEMENTO MURCIANO-ALMERIENSE
}

\author{
Juan LORITE MORENO, Francisco Bruno NAVARRO REYES \\ y Francisco VALLE TENDERO
}

New records for the vascular flora of Sierra Nevada (Almería): The Murciano-almeriense element.

Palabras clave. Flora, Sierra Nevada, Almería, Murciano-almeriense.

Key Words. Flora, Sierra Nevada, Almería, Murciano-almeriense.

Como resultado de los trabajos que estamos realizando sobre flora y vegetación de la Sierra Nevada Almeriense, se aportan citas de trece especies nuevas para el catálogo florístico de Sierra Nevada. Estas citas suponen un importante contingente murcianoalmeriense que caracteriza florísticamente la zona suroriental del parque. Si tenemos en cuenta que muchos de ellos son endemismos de área bastante restringida, o cuando menos 